\title{
Pediatric Pancreatic Lymphadenitis Tuberculosis Causing Inferior Vena Cava Thrombosis in Syria
}

\author{
Mhd Kutaiba Albuni ${ }^{*}$, Ruba Zuhri Yafi², Bana Sabbagh ${ }^{1}$, Mazen Al Moubarak ${ }^{3}$, Lina Khouri ${ }^{4}$ \\ ${ }^{1}$ Department of Internal Medicine, Al-Mouwasat University Hospital, Al Mazzeh, Damascus, Syria \\ ${ }^{2}$ Faculty of Medicine, Damascus University, Damascus, Syria \\ ${ }^{3}$ Department of Surgery, Damascus Hospital, Damascus, Syria \\ ${ }^{4}$ Department of Pediatrics, Faculty of Medicine, Damascus University, Damascus, Syria \\ Email: ^mhd.kutaiba@gmail.com
}

How to cite this paper: Albuni, M.K., Yafi, R.Z., Sabbagh, B., Al Moubarak, M. and Khouri, L. (2021) Pediatric Pancreatic Lymphadenitis Tuberculosis Causing Inferior Vena Cava Thrombosis in Syria. Journal of Tuberculosis Research, 9, 117-123.

https://doi.org/10.4236/jtr.2021.93011

Received: May 3, 2021

Accepted: July 5, 2021

Published: July 8, 2021

Copyright $\odot 2021$ by author(s) and Scientific Research Publishing Inc. This work is licensed under the Creative Commons Attribution International License (CC BY 4.0).

http://creativecommons.org/licenses/by/4.0/

\begin{abstract}
Background: Pancreatic tuberculosis (TB) is very rare, mostly due to the antibacterial effects of the pancreatic enzymes. The association of thrombosis and tuberculosis has been reported but that of inferior vena cava (IVC) thrombosis and pancreatic tuberculosis is extremely rare and has only been reported once. Case presentation: A case of pancreatic Tuberculosis and IVC thrombosis presented with constitutional symptoms. Ultrasonography and computerized tomography showed a lesion in the head of the pancreas and a large lymph mass. Magnetic resonance imaging (MRI) of (IVC) showed thrombosis in the IVC. Histological examination revealed necrotizing granulomas after a laparotomy. The patient received antituberculosis chemotherapy and low molecular weight heparin. Conclusion: Pancreatic TB is a challenge to diagnose especially in Resource-poor countries, which might lead to delay in treatment and worse complications.
\end{abstract}

\section{Keywords}

Pancreas, Tuberculosis, Thrombosis, Inferior Vena Cava, Case Report

\section{Introduction}

Tuberculosis is a widespread disease, which is encountered in developing countries that still hold a great burden on public health. Pancreatic and peripancreatic TB is an exceedingly uncommon disease [1], but recently there is an increase in reports of pancreatic TB presenting as a hypoechoic mass on ultrasonography and imaging mimicking malignancy [2]. 
The exact pathogenesis is not proved yet but theories include Milliary TB, Hematogenous spread from the lungs, or in rare occasions from direct spread from neighboring lymph nodes [2]. The clinical presentation varies widely as there are no specific findings to point at the diagnosis [2]. Most cases are diagnosed after tissue biopsy or exploratory laparotomy, which makes the diagnosis even more challenging in resource-poor hospitals like in Syria.

The association of $\mathrm{Tb}$ and thrombosis of inferior vena cava (IVC) is extremely rare and has been only reported once [3]. Here we present a case of Pancreatic TB with IVC diagnosed after a laparotomy.

\section{Case Presentation}

A 9-year-old boy without significant history was admitted to the pediatric hospital for fatigue, weakness, unmeasured weight loss, night sweats, loss of appetite, generalized abdominal pain, and vomiting. Physical examination revealed a pale jaundiced child, and swollen occipital, submandibular and inguinal lymph nodes which move easily, there was also abdominal distension and 39.5C fever.

The laboratory test detected low mean corpuscular volume (MCV) anemia with hemoglobin: $7.4 \mathrm{~g} / \mathrm{dl}$, WBC count of $19,000 / \mathrm{mm}^{3}$ with $78 \%$ of the cells being mature neutrophils, chronic reactive protein (CRP) was $99.5 \mathrm{mg} / \mathrm{l}$. Liver enzymes were: Gamma Glutamyl Transpeptidase (GGT) 1104 U/L, Aspartate Aminotransferase (AST) 262.9 U/L, and Alanine Aminotransferase (ALT) 314.0 U/L. Total Bilirubin (TB) $3.2 \mathrm{mg} / \mathrm{dl}$ and Direct Bilirubin (DB) $2.5 \mathrm{mg} / \mathrm{dl}$. Tuberculin skin test (Mantoux tuberculin test) was negative.

Labs are summarized in (Table 1).

Abdominal ultrasound revealed bile duct expansion of $5 \mathrm{~cm}$ at the hepatic hilum, hypo-echoic and heterogeneous mass measuring $5 \mathrm{~cm}$ in the head of the pancreas, and pancreatic duct measuring $2.5 \mathrm{~mm}$ with a large amount of fluid in the pelvis, lymph nodes enlargement at the hepatic hilum measuring $3 \mathrm{~cm}$.

The patient underwent computed tomography of the abdomen, which revealed signs of thrombosis in (IVC) (Figure 1). Moreover, magnetic resonance imaging (MRI) of the abdomen showed a large lymph mass surrounding the superior mesenteric artery extending to the liver hilum and pancreatic head (Figure 2), next

Table 1. Summarize the patient's labs.

\begin{tabular}{cc}
\hline WBC (White Blood cell count) & $19,000 / \mathrm{mm}^{3}$ \\
Neutrophils & $78 \%$ \\
Chronic Reactive Protein (CRP) & $99 \mathrm{Mg} / \mathrm{L}$ \\
Aspartate Amino Transferase (AST) & $262 \mathrm{IU} / \mathrm{L}$ \\
Alanine Amino Transferase (ALT) & $314 \mathrm{IU} / \mathrm{L}$ \\
Gamma Glutamyl Transpeptidase (GGT) & $1104 \mathrm{IU} / \mathrm{L}$ \\
Total Bilirubin (TB) & $3.2 \mathrm{mg} / \mathrm{dl}$ \\
Direct Bilirubin (DB) & $2.5 \mathrm{mg} / \mathrm{dl}$ \\
\hline
\end{tabular}




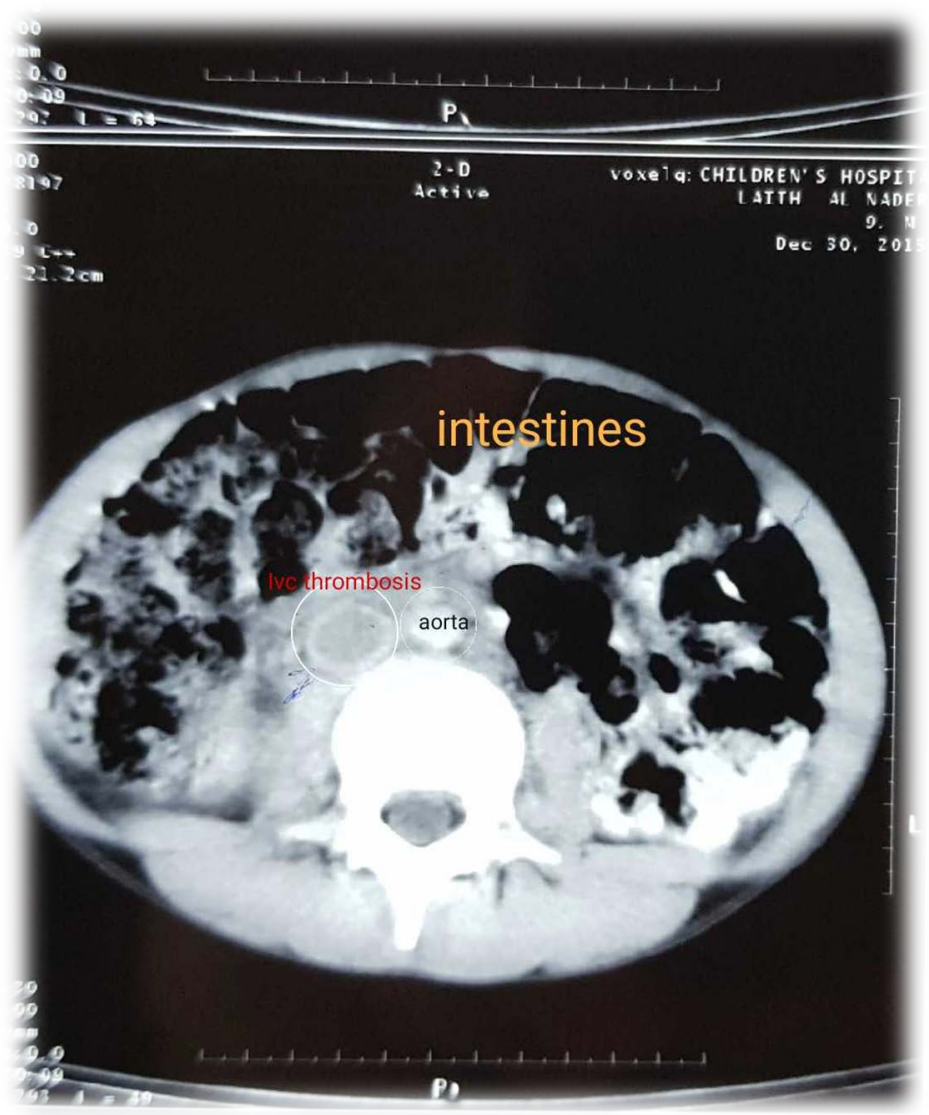

Figure 1. CT scan of the abdomen shows signs of thrombosis in the IVC.

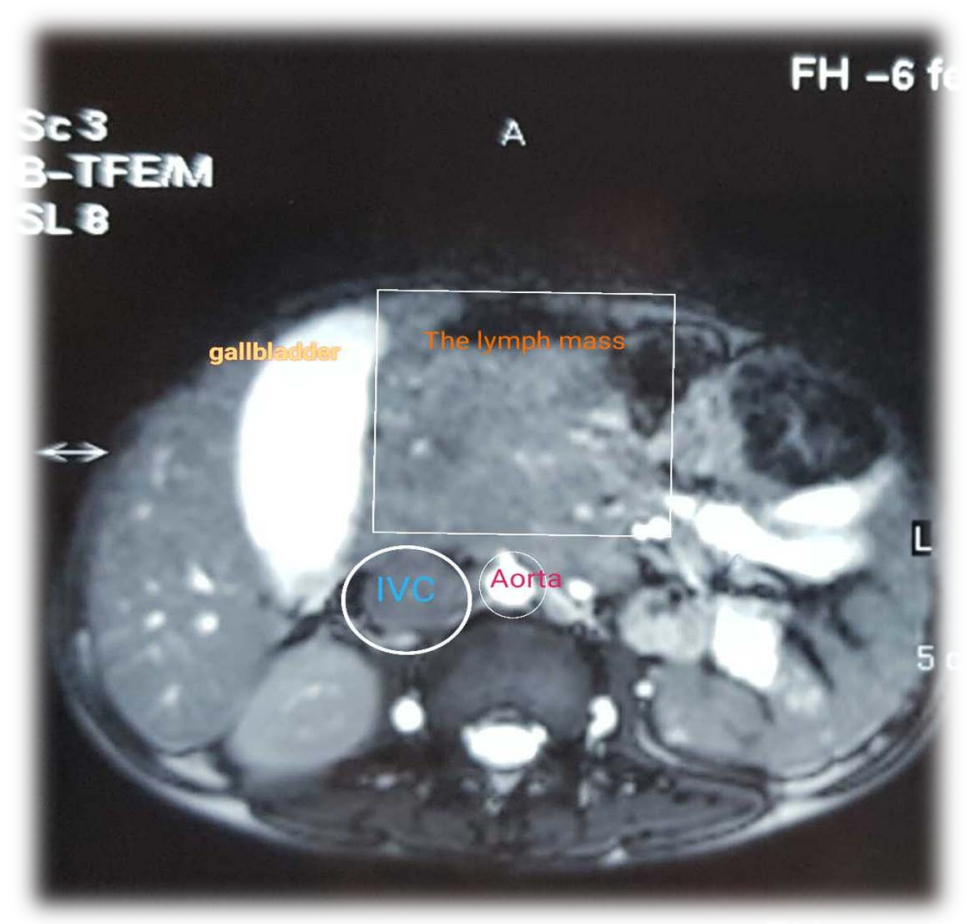

Figure 2. MRI of the abdomen shows a large lymph mass extending to the liver hilum and pancreatic head. 


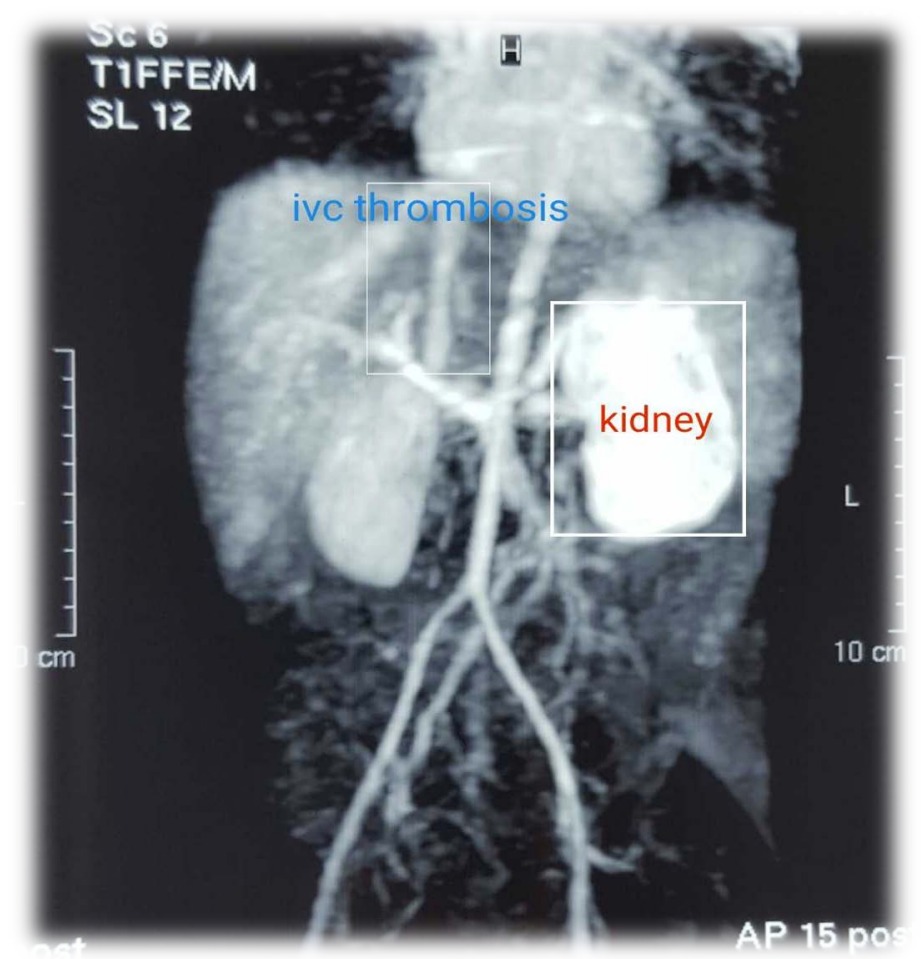

Figure 3. MR angiography of the IVC shows thrombosis in the IVC starts above the renal vein and down to the pelvis.

we did magnetic resonance angiography of inferior vena cava (IVC) which showed thrombosis in the IVC starting above renal vein and down to the pelvis (Figure 3).

Due to the lack of resources, we couldn't do CT guided biopsy, so the patient underwent an exploratory laparotomy and we took biopsies from the head, the body of the pancreas, and mesenteric lymph nodes. Microscopically, there were necrotizing granulomas consistent with TB in the body of the pancreas, nonspecific pancreatitis, and granulomatous lymphadenitis compatible with TB in mesenteric lymph nodes. TB treatment was started in addition to low molecular weight heparin (Clexane). The patient was discharged safely on anti TB medications for six months with follow-up in the clinic, unfortunately, due to the ongoing crisis in Syria back then we lost the follow-up with the patient.

\section{Discussion}

TB continues to load a huge burden on health in the middle east with an incidence of 115 per 1,000,000 [4]. Abdominal Tuberculosis is the most common extrapulmonary manifestation that includes a variety of intraabdominal organs like intestines, gastroduodenal, liver, biliary tract, spleen, and pancreas [5].

Pancreatic TB with or without lymph node involvement is a rare phenomenon, as it accounts only for $4.7 \%$ of all locations [1]. This rare occurrence of pancreatic TB is mostly attributed to the antibacterial effects of pancreatic lipases, and deoxyribonucleases [2]. 
Pancreatic TB presents with a variety of symptoms such as anorexia, weight loss, night sweat, fever, and obstructive jaundice [2] [5]. The vague presentation can cause confusion as it can mimic the classic scenario of cancer [2]. In a study from Bangladesh, five of the 60 patients who had unresectable pancreatic cancer and underwent further evaluation for pancreatic malignancy were found to have pancreatic tuberculosis [5]. The differential diagnosis may also include pancreatic cystic neoplasms, chronic pancreatitis, and autoimmune pancreatitis [5].

In general, the diagnosis of pancreatic $\mathrm{TB}$ requires a high degree of suspicion but being in an endemic area of TB like Syria usually puts TB in the differential diagnosis. The first step in approaching our patient was to do an abdominal ultrasound that revealed a hypoechoic lesion which is the most common finding in pancreatic TB [5], next we did a computed tomography of the chest and abdomen that showed beside the pancreatic lesion a large lymph mass surrounding the superior mesenteric artery extending to the liver hilum and pancreatic head. Although there are no definitive features of pancreatic TB on CT scan [5], This presence of peripancreatic adenopathy made us think more of a TB origin of this lesion, therefore the patient underwent a laparotomy and the histological examination of the pancreatic lesion and the lymph nodes showed necrotizing granulomas. The presence of granulomas is the most common histological finding in $\mathrm{TB}$, on the other hand, the presence of positive $\mathrm{AFB}$ and culture was relatively low, and the use of RT-PCR was not yet well established to exclude TB [5], thus we initiated a trial of antituberculosis therapy, and the patient responded clinically which supported our diagnosis.

An association between $\mathrm{Tb}$ and thrombosis was established before, for example, In the MEDENOX study, patients with TB were found to have a greater risk of venous thromboembolism, with an odds ratio of 1.62 [6]. Moreover, Several case reports mentioned an association between TB and venous thrombosis [7] [8]. In one retrospective analysis of TB patients in Seoul from 2000 to 2015, there was a higher incidence of thromboembolism than the general population, and the vast majority were associated with Pulmonary $\mathrm{Tb}$ [9]. However, that of pancreatic TB and inferior vena cava thrombosis is extraordinary and has been only reported once [3].

The exact mechanism of thrombosis and TB has not yet been established, one hypothesis suggests the role of proinflammatory cytokines (interleukin 1, interleukin 6, tumor necrosis factor $\alpha$ ) [10], another hypothesis says that thrombosis originate from local factors like compression from neighboring adenopathy [11] another mechanism suggests that inflammation caused by TB in the lower respiratory system leads to fibrin deposition that causes a hypercoagulation state that may persist for 2 weeks.

\section{Conclusion}

Pancreatic TB is a very rare disease that opposes a big challenge to diagnose especially in Resource-poor countries, which might lead to delay in treatment and 
worse complications. The association between TB and thromboembolism has been established and requires more attention regarding prophylactic anticoagulation.

\section{Declarations}

\section{Ethics Approval and Consent to Participate}

The authors declared that there was consent approval to participate

\section{Consent for Publication}

Written informed consent was obtained from the patient's guardian for publication of this case report and any accompanying images

\section{Availability of Data and Material}

Available.

\section{Authors' Contributions}

M.K.A, R.Y, B.S, M.A, L.K contributed equally read and approved the manuscript.

\section{Conflicts of Interest}

The authors declare no conflicts of interest regarding the publication of this paper.

\section{References}

[1] Uygur-Bayramiçli, O., Dabak, G. and Dabak, R. (2003) A Clinical Dilemma: Abdominal Tuberculosis. World Journal of Gastroenterology, 9, 1098-1101.

https://doi.org/10.3748/wig.v9.i5.1098

[2] Nagar, A.M., Raut, A.A., Morani, A.C., Sanghvi, D.A., Desai, C.S. and Thapar, VB. (2009) Pancreatic Tuberculosis: A Clinical and Imaging Review of 32 Cases. Journal of Computer Assisted Tomography, 33, 136-141. https://doi.org/10.1097/RCT.0b013e31816c82bc

[3] Laamrani, F.Z. and Dafiri, R. (2014) A Rare Pediatric Case of Pancreatic Tuberculosis with Veinous Thrombosis. Diagnostic and Interventional Imaging, 95, 455-456. https://doi.org/10.1016/j.diii.2013.12.022

[4] WHO (2019) Global Tuberculosis Report 2019.

[5] Sharma, V., Rana, S.S., Kumar, A. and Bhasin, D.K. Pancreatic Tuberculosis. Journal of Gastroenterology and Hepatology, 31, 310-318. https://doi.org/10.1111/jgh.13174

[6] Alikhan, R., Cohen, A.T., Combe, S., Samama, M.M., Desjardins, L., Eldor, A., et al. (2004) Risk Factors for Venous Thromboembolism in Hospitalized Patients with Acute Medical Illness: Analysis of the MEDENOX Study. Archives of Internal Medicine, 164, 963-968. https://doi.org/10.1001/archinte.164.9.963

[7] Fullerton, D., Shrivastava, A., Munavvar, M., Jain, S., Howells, J. and Macdowall, P. (2007) Pulmonary Tuberculosis Presenting with Central Retinal Vein Occlusion. British Journal of Ophthalmology, 91, 1714-1715. https://doi.org/10.1136/bjo.2007.114777 
[8] Gogna, A., Grover, S.B., Arun, S. and Saluja, S. (2004) Isolated Hepatic Inferior Vena Cava Thrombosis in a Case of Tuberculosis. Indian Academy of Clinical Medicine, 5, 267.

[9] Ha, H., Kim, K.H., Park, J.H., Lee, J.K., Heo, E.Y., Kim, J.S., Kim, D.K., Choi, I.S., Chung, H.S. and Lim, H.J. (2019) Thromboembolism in Mycobacterium tuberculosis Infection: Analysis and Literature Review. Infection \& Chemotherapy, 51, 142 149. https://doi.org/10.3947/ic.2019.51.2.142

[10] Hussain, R., Shiratsuchi, H., Phillips, M., Ellner, J. and Wallis, R.S. (2001) Opsonizing Antibodies (IgG1) Up-Regulate Monocyte Proinflammatory Cytokines Tumour Necrosis Factor-Alpha (TNF- $\alpha$ ) and IL-6 But Not Anti-Inflammatory Cytokine IL-10 in Mycobacterial Antigen-Stimulated Monocytes-Implications for Pathogenesis. The Journal of Transitional Immunology, 123, 210-218.

https://doi.org/10.1046/j.1365-2249.2001.01439.x

[11] Gogna, A., Pradhan, G., Sinha, R. and Gupta, B. (1999) Tuberculosis Presenting as Deep Vein Thrombosis. Postgraduate Medical Journal (PM), 75, 104-106.

https://doi.org/10.1136/pgmj.75.880.104

\section{Abbreviations}

TB: Tuberculosis

CT scan: Computed tomography scan

MRI: Magnetic resonance imaging

IVC: Inferior vena cava. 